\title{
REVIEW
}

\section{Morphologic and Molecular Features of Breast Ductal Carcinoma in Situ}

Souzan Sanati

From the Department of Pathology and Immunology, Washington University in St Louis, St. Louis, Missouri

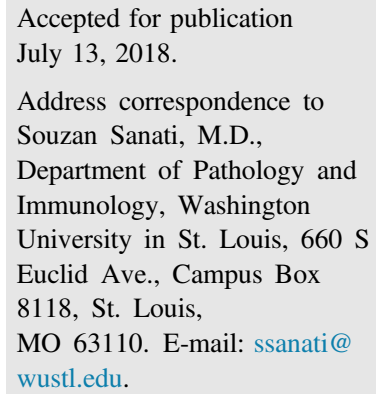

\begin{abstract}
Ductal carcinoma in situ (DCIS) encompasses a highly heterogeneous group of lesions that differ with regard to their clinical presentations, histologic features, biomarker profiles, genetic abnormalities, and potential for progression. DCIS is a nonobligatory precursor for invasive carcinoma. With the advent of screening mammography, the incidence of DCIS has significantly increased. There is an argument that many of these lesions will not progress to invasive carcinoma within the lifetime of a patient. In addition, many studies have found enormous heterogeneity within DCIS. There is a need for biomarkers that can stratify patients with DCIS into different prognostic groups based on the biology of the disease. Estrogen and progesterone receptors are the established biomarkers that are used for clinical decision making. In addition, a number of biomarkers, such as human epidermal growth factor receptor 2 protein, $p 53$ tumor suppressor gene, Ki-67 proliferation marker, and tumor-infiltrating lymphocytes, carry prognostic significance, although their use is considered investigational and has not been transferred to clinical practice. On the molecular level, low-grade and high-grade DCIS have different molecular alterations, and the intrinsic molecular subtypes that exist in invasive carcinoma also exist in DCIS with prognostic implications. In this article, we review the morphologic features, prognostic biomarkers, and molecular features of DCIS. (Am J Pathol 2019, 189: 946-955; https://doi.org/ 10.1016/j.ajpath.2018.07.031)
\end{abstract}

The term ductal carcinoma in situ (DCIS) encompasses a highly heterogeneous group of lesions that differ with regard to their clinical presentations, histologic features, biomarker profiles, genetic abnormalities, and potential for progression. They have in common a monoclonal proliferation of neoplastic epithelial cells confined to the terminal ductallobular units (TDLUs; the smallest terminal branches of the ductal system) and typically grow in a segmental distribution without extension through the basement membrane. ${ }^{1}$ DCIS may grow into larger ducts or into adjacent lobules, giving an appearance referred to as cancerization of lobules. ${ }^{1,2}$ DCIS is a nonobligatory precursor for invasive carcinoma. Diagnosis of DCIS indicates a high risk of subsequent breast cancer at the index breast or near the site of prior DCIS. ${ }^{3}$ The evidence that DCIS is a precursor of invasive carcinoma is indirect but convincing: invasive carcinoma is rarely seen without DCIS, women who are diagnosed with DCIS have a 10 times higher risk of development of ipsilateral invasive carcinoma if left untreated, DCIS and invasive carcinoma share the same genetic and molecular features, and microscopic examination reveals continuity between DCIS and invasive carcinoma. ${ }^{4}$

Before widespread use of screening mammography, DCIS was an incidental pathologic finding in more advanced, palpable tumors. With the advent of screening mammography, DCIS is now diagnosed mostly through screening-detected calcifications or architectural abnormalities, and its incidence has increased from 5\% in the premammographic era to $20 \%$ to $25 \%$ of breast cancer. ${ }^{2,5}$ The main concern in the management of DCIS is prevention of disease recurrence, mainly as invasive carcinoma, which constitutes $50 \%$ of disease recurrences. ${ }^{5}$ It has been argued that higher prevalence of diagnosed DCIS has

\footnotetext{
Disclosures: S.S. is a consultant for Genentech, Inc.

This article is part of a review series on Ductal Carcinoma in Situ-Discerning Aggressive versus Benign Disease Using Molecular
} Features. 
resulted in diagnosing indolent lesions that would not result in an invasive recurrence during the lifetime of the patient. This theory is also supported by the high prevalence of DCIS in autopsy cases of patients who died of other causes. ${ }^{6,7}$ Although this may be true because only $30 \%$ of DCIS will have an invasive recurrence, ${ }^{8}$ there are no known biomarkers that can reliably identify DCIS with potential to progression. The result is a potential overdiagnosis and overtreatment in a large number of patients with DCIS. A consensus conference at the National Institute of Health State of the Science conference in 2009 has recommended eliminating the term carcinoma for these lesions. ${ }^{9}$ Other experts have proposed using the term borderline breast lesions to avoid overdiagnosis and more importantly overtreatment in these lesions with indolent biology. ${ }^{10}$ These terms also take into consideration the high interobserver variability in diagnosing these lesions. The overdiagnosis rate is approximately $61 \%$ for low-grade (LG), $57 \%$ for intermediate grade (IG), and $45 \%$ for high-grade (HG) lesions. ${ }^{8}$ In this article, we review morphologic features of DCIS with a discussion of its common differential diagnoses and molecular features.

\section{Morphologic Features of DCIS}

Most cases of DCIS are not grossly visible. If palpable, DCIS may form a mass with cords of tissue extruding a pastelike material from their cut surfaces (a gross correlation to the material seen on histologic sections as comedo necrosis). DCIS is morphologically seen as proliferation of neoplastic cells with variable nuclear cytologic features and in a variety of growth patterns that usually grow over and obliterate the luminal spaces of the ducts and TDLUs. HG-DCIS can incite a desmoplastic stromal response mixed with chronic inflammation, which may raise the concern for invasive carcinoma (Figure 1). Myoepithelial markers may be useful in such cases because they highlight the myoepithelial layer, thus providing evidence that proliferation has not yet breached the TDLUs. Overall, it is recommended not to rely on only one myoepithelial marker. Combination of a nuclear (such as p63) and a cytoplasmic (such as smooth muscle myosin heavy chain or calponin) marker is recommended for the best results. ${ }^{11}$ DCIS

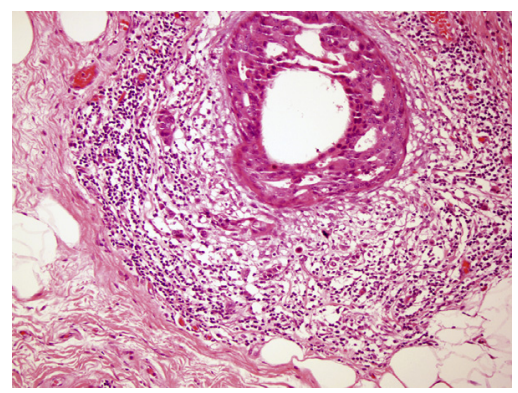

Figure 1 High-grade ductal carcinoma in situ with a desmoplastic stromal response and mixed chronic inflammation. Notice the single cells and small clusters of atypical cells within the dense inflammatory background, suggestive of microinvasion. Hematoxylin and eosin stain was used. Original magnification, $\times 20$.

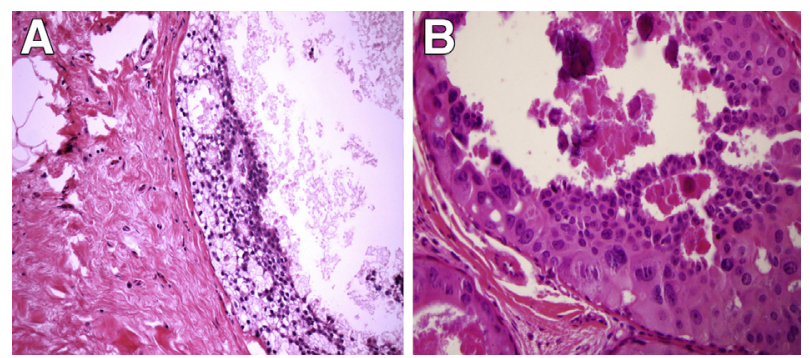

Figure 2 A: Ductal carcinoma in situ with clear cell features. B: Highgrade ductal carcinoma in situ with apocrine features. Hematoxylin and eosin stain was used (A and $\mathbf{B})$. Original magnification, $\times 20(\mathbf{A}$ and $\mathbf{B})$.

may become displaced during previous needle biopsy, leading to carcinoma within a biopsy tract that may be confused with invasive breast carcinoma (IBC) in the subsequent excisional specimen. DCIS may have a variety of morphologic features, including clear cell and apocrine differentiation (Figure 2). Although familiarity with these morphologic patterns is helpful in recognizing the lesion, most of these morphologic variants do not bear clinical significance independent of their nuclear grade.

For clinical purposes, a few prognostic and/or diagnostic characteristics of in situ carcinomas should be reported in a pathology report. This information may affect the clinical decision making.

\section{Estimate of Size and Extent}

If the lesion is only present on one slide, microscopic measurement of the focus of DCIS will be the most accurate measurement. If the lesion is present on multiple slides, the most accurate measurement is achieved by correlating the microscopic sections that contain DCIS, with a gross diagram of the breast specimen indicating location and relationship of different sections within the specimen. By correlating the two, an estimate size of the lesion can be provided. In rare cases where an accurate size estimate cannot be given, extent of the lesion can be reported as the fraction of slides involved by DCIS.

\section{Nuclear Grade}

Nuclear grade has emerged as a strong predictor of local recurrence for DCIS. ${ }^{5}$ On the basis of nuclear features, including nuclear size, polarization, presence and size of nucleoli, and mitotic figures, DCIS is classified into 3 grades: LG, IG, or HG. ${ }^{1,6}$ LG-DCIS consists of a proliferation of small, monomorphic, and evenly spaced luminal epithelial cells with homogeneous chromatin distribution and inconspicuous nucleoli. The neoplastic cell nuclei approximate that of normal ductal epithelial cells. Mitotic counts are usually low. Small laminated calcifications are common. ${ }^{4}$ In contrast, HG-DCIS consists of a proliferation of large, highly pleomorphic, variably spaced, less organized epithelial cells with vesicular or coarse chromatin, irregular nuclear contours, and prominent or multiple nucleoli. The neoplastic cells 
can have up to three times the variation in nuclear size. Mitotic counts are high, apoptotic cells are frequent, and comedo necrosis (necrosis in the center of the epithelial proliferation) is common. Amorphous calcifications associated with necrosis are common. ${ }^{4}$ IG carcinomas are lesions at the overlapping ends of LG- and HG-DCIS with features that fall between the two categories. ${ }^{1,5}$ Studies have found that the IG lesions have the highest interobserver variability. ${ }^{12}$ As with invasive carcinomas, DCIS commonly has significant heterogeneity with areas of multiple histologic grades. ${ }^{13}$

\section{Growth Pattern}

DCIS is extremely diverse histologically, with respect to both nuclear grade and architecture. A variety of different growth patterns can be recognized, although commonly more than one growth pattern is present within a given lesion. Familiarity with these growth patterns is necessary for recognition of DCIS. The most common growth patterns include solid, papillary, micropapillary, and cribriform (sievelike) architectural patterns (Figure 3).

In the cribriform growth pattern, the neoplastic cells form punched-out spaces with round and rigid extracellular lumens formed by neoplastic cells polarizing around the spaces. LGDCIS with cribriform growth pattern should be differentiated from collagenous spherulosis, particularly when collagenous spherulosis is involved by lobular carcinoma in situ. The spaces in collagenous spherulosis are lined by myoepithelial cells with spindled nuclei (which can be highlighted by myoepithelial markers), and the round spaces are filled by basement membrane material. In cases in which collagenous spherulosis is associated with lobular carcinoma in situ, an E-cadherin stain can highlight the lobular nature of neoplastic cells. ${ }^{14}$ Adenoid cystic carcinoma occasionally can be mistaken for cribriform DCIS, but adenoid cystic carcinoma is composed of combined epithelial and myoepithelial cells
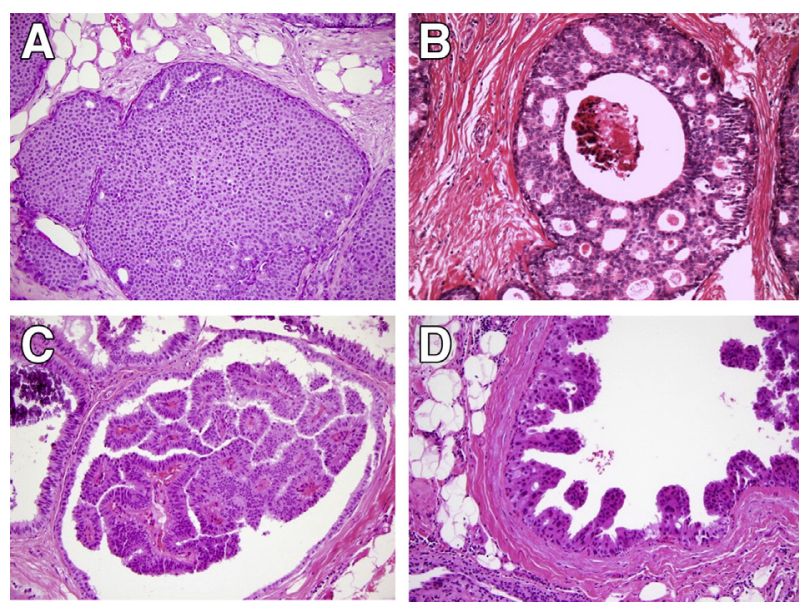

Figure 3 A: Ductal carcinoma in situ with solid growth pattern. B: Ductal carcinoma in situ with cribriform growth pattern and central comedo necrosis. C: Ductal carcinoma in situ with papillary growth pattern. D: Ductal carcinoma in situ with micropapillary growth pattern. Hematoxylin and eosin stain was used (A-D). Original magnification, $\times 20(\mathbf{A}-\mathbf{D})$. with basement membrane material and has an infiltrative growth pattern, whereas cribriform DCIS has a monomorphic appearance with epithelial cells polarized surrounding the cribriform spaces. A panel of stains, including myoepithelial markers, estrogen receptor (ER), c-kit, and MYB, can aide in the differential diagnosis. ${ }^{14}$ Invasive cribriform carcinoma has sievelike irregular contours and an infiltrative growth pattern. Myoepithelial markers can confirm the absence of myoepithelial cells surrounding the cellular proliferations.

The micropapillary growth pattern is manifested by a proliferation of tufts of neoplastic cells that project into the lumen of TDLUs. These tufts are characterized by a lack of fibrovascular cores, a club-shaped end, and proliferation of a monomorphic, evenly distributed cell population. ${ }^{1}$ Usual ductal hyperplasia can have a micropapillary growth pattern. The micropapillations of usual ductal hyperplasia have a broad base and a narrow pinched tip and are composed of cells that show larger nuclei at the base and appear smaller at the tip. This is in contrast with micropapillary DCIS, which has a narrow base and a broad bulbous tip and is composed of nuclei that are enlarged throughout the micropapillae. ${ }^{14}$

The solid growth pattern is characterized by a proliferation of sheets of monomorphic, cohesive cells that fill the lumen of TDLUs. These cells may form pseudorossettes or microacini with polarization of surrounding cells.

Papillary DCIS consists of a papillary proliferation characterized by branching fibrvascular cores covered by a monotonous epithelial proliferation with mild to moderate nuclear atypia. In contrast to benign papillomas, papillary DCIS has no invested myoepithelial layer, as indicated by a lack of staining with myoepithelial markers, such as p63 and calponin. Some markers, such as smooth muscle actin can highlight the myoepithelial cells, but they can also stain the vascular walls within the fibrovascular cores of papillary DCIS, which should not be overinterpreted as evidence of a myoepithelial layer.

The clinging growth pattern is recognized by some experts as a distinct growth pattern. It is characterized by a duct lined by one or a few layers of highly atypical neoplastic cells at the periphery and filled by abundant comedonecrosis. Some experts consider this growth pattern a variation of the solid growth pattern with extensive necrosis.

\section{Extent of Comedo Necrosis}

Comedo necrosis is usually seen in IG- and HG-DCIS. It is usually calcified necrosis within the center of a duct that is associated with DCIS. Some studies have found that abundant comedo necrosis is associated with increased local recurrence after lumpectomy for DCIS.

\section{Paget Disease of the Nipple}

Skin involvement (Paget disease) of the nipple can be seen when neoplastic cells percolate through the underlying lactiferous ducts to the epithelium without breaching the basement membrane. This condition is usually associated with 
underlying HG-DCIS. It is important to distinguish the Paget cells within nipple squamous epithelium from other clear cells that occasionally can be seen within the nipple, mainly Toker cells. Toker cells are incidentally found clear cells within the nipple, which are smaller compared with Paget cells and do not have significant atypia. A human epidermal growth factor receptor 2 (HER2) immunohistochemical stain can be useful in difficult cases because the cells of Paget disease are positive for HER2, whereas Toker cells are negative. ${ }^{15}$

\section{Surgical Margins}

Surgical margin status is of particular importance and has an inverse association with the incidence of local recurrence. Because DCIS usually is not grossly visible, margins should be microscopically examined, and the location of the closest margin and its distance from DCIS should be mentioned in the report. A recent meta-analysis and consensus statement indicated that for patients with pure DCIS who will undergo whole breast radiation, a margin of $\geq 2 \mathrm{~mm}$ will be considered adequate. Patients with foci of DCIS within $2 \mathrm{~mm}$ from an inked margin may require additional excision, but multiple other patient- and tumor-related factors may affect the decision for further surgery. ${ }^{14,16}$ The 2-mm distance from the margin was chosen to account for skip lesions and discontinuity of DCIS in the ductal system. ${ }^{17}$ Other studies have questioned the validity of the 2-mm margin requirement. ${ }^{18}$ The extent of involved margin (focal, multifocal, extensive) should be reported. The presence of associated microcalcifications should be noted because DCIS that is first detected by microcalcifications will frequently recur with microcalcifications.

\section{Diagnosis on Core Biopsy Material}

Atypical ductal hyperplasia has significant morphologic overlap with LG-DCIS. The distinction between DCIS and atypical ductal hyperplasia is usually based on quantitative ( $\geq 2 \mathrm{~mm}$ or $>2$ duct spaces) or qualitative criteria. The widespread use of screening mammography has resulted in targeting smaller lesions on core biopsies. It is recommended that only lesions with unequivocal features of DCIS be diagnosed as such on core biopsy and DCIS classification of limited volume of disease with borderline morphologic findings be deferred to full examination of the extent of the disease on surgical specimen.

Diagnosis of DCIS on core biopsy should prompt a surgical excision with clear margins. Excision is usually performed to preserve breast tissue. A mastectomy is performed only when achieving clear margins cannot be obtained by conservative surgery, for example, in patients with extensive, multifocal DCIS or in patients who cannot receive radiotherapy. ${ }^{5}$ Sentinel lymph node biopsy generally is not performed unless there is a high risk of invasive cancer (large lesions, HG-DCIS) or in patients who will undergo mastectomy. An excision in most patients will be followed by adjuvant radiotherapy. Adjuvant radiotherapy reduces the recurrence rate of DCIS by $50 \%$, regardless of nuclear grade. ${ }^{19}$ There are ongoing efforts to identify a group of patients who have such low risk of recurrence or progression who will not benefit substantially from radiotherapy; however, efforts in identifying biomarkers for such a low-risk group of patients have not yet been successful. ${ }^{14}$ Patients with hormone receptor-positive DCIS may further benefit from administration of tamoxifen. ${ }^{3,20}$ The National Surgical Adjuvant Breast and Bowel Project B-24 trial evaluated 732 patients with DCIS without any knowledge of their biomarker status. Tamoxifen was administered to all patients. ER was retrospectively evaluated, and response to treatment was correlated with the degree of ER expression. The study found significant risk reduction in subsequent breast cancer recurrence in 10 years of follow-up in patients who had ER-positive DCIS. ${ }^{20}$ The hormone receptor-negative patients did not benefit from tamoxifen.

A variety of clinical and histopathologic factors have been used as prognostic markers in DCIS. These factors include young patient age, ${ }^{21}$ mode of presentation (mass versus mammographic abnormality), extent of DCIS, nuclear grade, comedo necrosis, multifocality, and margin status. Nuclear grade and comedo necrosis are the strongest pathologic prognostic factors. ${ }^{5,22}$ Long-term follow-up of $>800$ patients with DCIS in UK National Health Service Breast Screening Programme shows a shorter median interval to invasive recurrence in HG-DCIS (76 months) compared with LGDCIS (131 months). ${ }^{23}$

A combination of the above-mentioned prognostic factors has been used to stratify patients with DCIS into different risk groups. The University of Southern California/Van Nuys Prognostic Index was developed using nuclear grade, tumor size, margin width, comedo necrosis, and patient age..$^{5}$ The prognostic utility of the Van Nuys system was validated by some groups ${ }^{24}$ but not others. ${ }^{12}$ Other groups have developed nomograms based on a combination of clinicopathologic parameters (eg, Memorial-Sloan-Kettering Cancer Center nomogram ${ }^{25}$ with imperfect results. ${ }^{26}$ Inclusion of endocrine and molecular biomarkers in this nomogram has been suggested to improve its predictive value. ${ }^{27}$

Although these parameters are able to provide prognostic information, they are not able to accurately predict which patients will have disease recurrence. ${ }^{28}$ In addition, there is significant lack of reproducibility among pathologists; for example, assessment of nuclear features has found that up to $30 \%$ of cases in multicenter clinical trials required reclassification on central review. ${ }^{2}$ There is an unmet need for more objective methods for classification and risk stratification of DCIS. The main challenge in the clinical management of DCIS continues to be tailoring of treatment to individual risk to avoid overtreatment of LG lesions or undertreatment of HG lesions. ${ }^{29}$

\section{Prognostic and Predictive Biomarkers}

The role of biomarkers in establishing the prognosis and management of patients with breast cancer cannot be 
overemphasized. Established biomarkers, such as ER and progesterone receptors (PRs), are expressed in $70 \%$ to $80 \%$ of DCIS and have been used both as prognostic factors and predictors of response to endocrine therapy. ${ }^{12}$

Currently, evaluation of ER in patients with DCIS is considered standard of care and is mandated by National Comprehensive Cancer Network guidelines. ${ }^{28}$ The shift toward an earlier diagnosis of breast cancer because of improved imaging methods and screening programs highlights the need for further biomarker discovery to quantify the risk for individual patients. ${ }^{30}$

\section{ERs and PRs}

The ER belongs to a family of nuclear hormone receptors that function as transcription factors when they are bound to their respective ligands. ERs and PRs are parts of complex signaling pathways that interact with multiple survival and proliferation pathways in the cell and play a critical role in the development and progression of breast cancer. They have proven useful as prognostic factors, and more importantly as predictive factors, in the clinical management of breast cancer.

Assessment of ER expression by immunohistochemistry is an essential step in the evaluation of DCIS. Immunohistochemistry can be highly affected by a variety of preanalytic factors, including time of tissue fixation and the antigen retrieval method. ${ }^{31}$ The expression of PR is strongly dependent on the presence of ER and is reflective of a functioning ER pathway.

Several studies have found an excellent concordance rate between evaluation of biomarkers on needle core tissue and excisional specimen. ${ }^{32-34}$ To minimize the effect of preanalytic variables on test results, the American Society of Clinical Oncology/College of American Pathologists recommended using only $10 \%$ neutral buffered formalin as a fixative and controlling the formalin fixation time between 6 and 72 hours.

To standardize immunohistochemical evaluation, several scoring systems that incorporate both intensity and percentage of staining have been established. In general, similar to invasive carcinoma, to obtain benefit from hormonal treatment, a sample should demonstrate nuclear staining in at least $1 \%$ of tumor cells. ${ }^{20,31}$ For patients with a diagnosis of DCIS, ER can be assessed on core biopsy or excision; however, given that approximately $13 \%$ to $30 \%$ of cases diagnosed as DCIS on core biopsy will upgrade to invasive carcinoma on surgical excision, there is an economical argument that it is better to assess ER on excisional biopsy once it is known whether there is an invasive component. ${ }^{14}$ If biomarkers are performed on core biopsy and an invasive carcinoma is identified on excisional biopsy, additional evaluation of biomarkers on the excisional specimen with invasive carcinoma is warranted.

ER expression in DCIS is inversely related to the nuclear grade, ${ }^{12,35}$ with higher expression in LG-DCIS and lower expression in HG-DCIS. LG- and IG-DCIS are almost always ER positive, whereas HG-DCIS has ER positivity is $69 \%$ of cases. ${ }^{11}$

\section{HER2}

Receptor tyrosine-protein kinase erbB-2 is a member of the epidermal growth factor receptor (EGFR) family of tyrosine kinase proteins. It is a membranous protein that is expressed in all epithelial cells at low levels. The HER2/neu oncogene is involved in the regulation of cell proliferation, survival, motility, and invasion. HER2 expression has also been associated with promotion of carcinogenesis by maintaining and increasing cancer stem cells. In invasive carcinoma, HER2 amplification is an independent prognostic marker of poor outcome in the absence of adjuvant treatment and has been associated with an increased rate of metastasis, decreased time to recurrence, and decreased overall survival. HER2 is also a predictor of responsiveness to anthracyclinebased chemotherapy and trastuzumab. Immunohistochemistry (to evaluate protein overexpression) and in situ hybridization (to evaluate gene amplification) are the most common methods to evaluate HER2 status.

In DCIS, expression of HER2 occurs in a higher proportion of cases compared with invasive carcinoma (approximately 35\% versus 20\%). ${ }^{36}$ HER2 expression in DCIS is associated with high nuclear grade, comedo necrosis, myxoid stromal change, and increased risk of in situ and invasive recurrence, especially if the lesion has an elevated $\mathrm{Ki}-67$ index. ${ }^{12,28,37,38}$

HER 2 expression seems to be an early event in DCIS and is not necessarily related to its progression to IBC. ${ }^{36}$ In the United States, HER2 evaluation in DCIS is not recommended for clinical decision making.

\section{Tumor-Infiltrating Lymphocytes}

Assessment of tumor infiltrating lymphocytes (TILs) can provide important prognostic information in solid tumors. TILs are also a robust prognostic marker in IBC..$^{39}$ Assessment of TILs in DCIS has a strong correlation with ER-negative, HER2-overexpressing HG-DCIS. ${ }^{40,41}$ HGDCIS, especially if associated with comedo necrosis, has higher TILs. The comedo necrosis is related to the increased growth of the neoplastic cells with clonal expansion that surpasses the available blood supply. ${ }^{41,42}$ Stromal TILs also mirror the mutational load, fraction of genome altered, and the number of telomeric imbalances, which all cause neoantigen formation and evoke a immune response. ${ }^{41-43}$ TILs are significantly higher in DCIS with $p 53$ mutation. This could be confounded by the association of $p 53$ mutation with other features associated with TILs, such as high nuclear grade, ER negativity, and HER2 positivity. ${ }^{41,43}$ Stromal inflammation in DCIS diagnosed on core biopsy is a predictor of invasive cancer on subsequent excision. ${ }^{44}$

The method for TIL assessment has been previously published for solid tumors. ${ }^{39}$ Toss et $\mathrm{al}^{43}$ compared different methods for TIL assessment in DCIS and correlated the results with outcome. They concluded that TILs that touch 
neoplastic epithelial cells or at a distance of one lymphocyte from the epithelial cells had the highest correlation with outcome. Dense TILs were associated with shorter recurrence-free survival irrespective of ER status.

TILs in DCIS are dominated by $\mathrm{CD}^{+} \mathrm{T}$ cells, with $\mathrm{CD}^{+}{ }^{+}$helper $\mathrm{T}$ cells slightly outnumbering cytotoxic $\mathrm{CD} 8^{+}$ cells. If DCIS-associated TILs have lower T-cell to B-cell ratio, with a higher proportion of $\mathrm{FOXP}^{+}$regulatory $\mathrm{T}$ cells, the patient will have a higher recurrence rate. ${ }^{41,45,46}$ Because the data with respect to the clinical significance of the DCIS-associated immune response are rapidly expanding, pathologists may be challenged by providing this information in their reports in the future. ${ }^{47}$

\section{Molecular Features of DCIS}

There are three main models for the development of breast cancer. The first model was described by Wellings and Jensen $^{48}>30$ years ago. This model is a linear model of tumor evolution that describes progressive accumulation of random mutations from normal epithelium to atypical hyperplasia, carcinoma in situ, and ultimately invasive carcinoma. Wellings and Jensen ${ }^{48}$ proposed that the origin of most breast carcinomas is in the TDLUs. They described a continuous but nonobligatory evolution to invasive carcinoma through a series of increasingly abnormal phases during long periods. ${ }^{26}$ On the basis of this model, the transition from one stage to the next is primarily dependent on the accumulation of random genetic mutations.

The second model for breast cancer evolution, known as the branched model, is based on the evidence that LG- and HG-DCIS and invasive carcinomas have distinct molecular profiles; therefore, it is hypothesized that they evolve through distinct evolutionary pathways. ${ }^{2}$ LG-DCIS has lower number of alterations ${ }^{49}$; they are usually diploid or near diploid, express ER/PR, have $b c l 2$ mutation, and have recurrent loss of chromosome $16 q$ and gain of chromosome $1 q .{ }^{2}$ In contrast, HG-DCIS is usually aneuploid with complex genetic profiles. ${ }^{2} H E R 2$ amplification and EGFR mutation are common in HG-DCIS. ${ }^{49-53}$ Copy number gains in $1 q, 5 p, 8 q, 17 q$, and 20q; amplifications of $11 q 13,17 q 12$, and 17q22-24; and loss of $8 p, 11 q, 13 q$, and $14 q$ are characteristics of HG-DCIS. The p53 gene mutation, which is correlated with genomic instability, is present in approximately $40 \%$ of HG-DCIS. ${ }^{28,50,54}$ This model was later refined to distinguish the two pathways based on ER expression rather than grade and acknowledged the progression of some DCIS and invasive carcinomas from LG to HG. ${ }^{12}$

A third hypothesis is based on parallel progression of different grades of DCIS from a common progenitor stem cell diverging and progressing through different grades in parallel. These theories are all based on compelling observations and they may not be incompatible. ${ }^{13}$

The genomic events that determine DCIS occur long before morphologic evidence of disease. ${ }^{55}$ Recent studies have found that histologically normal-appearing breast epithelium is not always normal at the molecular level, and some of these morphologically silent genetic abnormalities may predispose the cells to premalignant or malignant transformation. ${ }^{56}$ The most significant alterations appear during transition from normal epithelium to DCIS. ${ }^{26,28}$ However, DCIS and invasive carcinoma are similar at the high resolution of global gene expression evaluated by microarrays, gene copy numbers, and promoter gene hypermethylation. $^{3,28}$

Carcinoma in situ is a late stage of tumor progression and the immediate precursor of invasive disease. ${ }^{28,56}$ DCIS has a continuum of histologically diverse proliferations that range from very well to very poorly differentiated, although, in clinical practice, they are often simply divided into two (eg, noncomedo versus comedo) or three (eg, LG versus IG versus HG) categories. This classification may be an oversimplification that fails to adequately convey their true diversity. ${ }^{56}$

A number of studies have found extensive heterogeneity and diversity in DCIS by assessment of morphologic features, protein expression, gene expression, and molecular alterations in a number of studies. Allred et $\mathrm{al}^{13}$ reported the extensive diversity in DCIS by a combination of morphologic evaluation, biomarker studies using immunohistochemistry, and molecular studies. They found broad distribution of different nuclear grades and biomarker profiles within a lesion. They also found that the magnitude of diversity in DCIS correlated with $p 53$ mutation, which was generally higher in HG-DCIS. King et $\mathrm{al}^{57}$ reported that most of their LG-DCIS developed HG invasive carcinoma and a substantial proportion of HG ER-positive cancers harbor genomic changes characteristic of LG-DCIS. Vincent-Salomon et $\mathrm{al}^{54}$ in a detailed morphologic and gene expression profiling study, found molecular heterogeneity in DCIS and a high degree of phenotypic, genomic, and transcriptomic similarities between DCIS and invasive ductal carcinoma. Using multiplex immunofluorescence, Gerdes et $\mathrm{al}^{58}$ reported that within DCIS there is significant heterogeneity among cells and cell clusters, different ducts, and patients.

These findings support that although earlier stages of carcinogenesis, such as flat epithelial atypia and atypical ductal hyperplasia, lack diversity and have LG morphologic and biological features, extensive diversity and heterogeneity occur at the stage of DCIS. They also support that at least a subgroup of HG ER-positive DCIS evolve from LG lesions because of accumulation of additional mutations. ${ }^{13,57}$ There is considerable plasticity within DCIS, and it is likely that regions with different characteristics compete for dominance, and eventually the most aggressive areas prevail. ${ }^{13}$

Interestingly, among clinicopathologic characteristics of DCIS, nuclear grade, more than any other pathologic feature, is correlated with number and pattern of molecular genetic abnormalities. ${ }^{51}$ The use of nuclear grade, however, is limited because of high interobserver variability among pathologists, which is particularly true for IG-DCIS. ${ }^{12,50}$ 


\section{Intrinsic Subtypes}

Gene expression profiling of IBC has categorized IBCs into luminal (luminal A and B) and nonluminal groups based on ER expression. The nonluminal group has been further classified into HER2 overexpressing and basal-like categories. Luminal A tumors differ from luminal B by higher ER expression and lower expression of proliferation-associated genes. ${ }^{59}$ This classification has major prognostic implications for IBC. Makretsov et $\mathrm{al}^{60}$ reported that it is possible to make the same categorization by IHC using markers that are aimed for luminal, HER2, and basal-like features. A number of studies have found that the same intrinsic molecular subtypes that exist in IBC are also identifiable in DCIS. ${ }^{13,54}$ Allred et $\mathrm{al}^{13}$ reported the enormous heterogeneity within every DCIS lesion, not only by nuclear grade and biomarker immunophenotype, but also by demonstrating multiple intrinsic molecular subtypes that coexisted in different regions of the same tumor. Others have applied immunohistochemistry surrogate markers to classify DCIS into intrinsic molecular subtypes. ${ }^{61}$ The luminal A group had high expression of ER, PR, and bcl2 and lacked HER 2 expression, and the HER2-overexpressing group was negative for ER, PR, and bcl2 and overexpressed HER $2 .{ }^{61}$ The distribution of molecular subtypes in DCIS using surrogate IHC markers has been reported as follows: luminal A, 55\% to $60 \%$; luminal B, $13 \%$; HER2 overexpressing, 14\%; and basal-like, $6 \%$ to $7 \%{ }^{54,62}$ The luminal A subgroup had the lowest recurrence rate $(7.6 \%)$ in 5 years of follow-up. ${ }^{36,63}$ The HER2overexpressing subtype had a higher prevalence in DCIS when compared with IBC, and the basal-like subtype (ER, PR, and HER2 negative and EGFR or CK5/6 positive) was relatively rare. ${ }^{28,63-65}$ Patients with luminal B (ER, PR, and HER2 positive) and HER2-overexpressing phenotypes had significantly higher risk of recurrence. ${ }^{17,29,63,66}$ There was a high correlation between HG-DCIS, HER2 overexpression, high Ki-67, and lack of ER and PR expression. ${ }^{24}$

The discovery of intrinsic molecular subtypes in DCIS has further validated DCIS as a precursor of different subtypes of invasive carcinoma, although these subtypes occur at different frequencies; for example, the frequency of the luminal B and HER2 subtypes is much higher in DCIS compared with IBC, and the frequency of the luminal A subtype is much higher in IBC compared with DCIS. ${ }^{28}$ In addition, the molecular subtypes correlate with grade; LG-DCIS is mostly composed of luminal phenotype (ER positive), and HG-DCIS has lower expression of ER, while expressing HER2 or basal-like phenotypes. Many studies have found prognostic value for classification of DCIS using surrogate markers by predicting overall and invasive recurrence. ${ }^{67}$

\section{Other Molecular Biomarkers}

In addition to the above-mentioned biomarkers, there is an increasing list of biomarkers that have the ability to provide prognostic information for DCIS. Ki-67 is a well-established marker of proliferating cells. It is a nuclear antigen and is expressed in all active phases of cell cycle but not in the resting cells (G0 phase). A meta-analysis of published literature found an association between high Ki-67 expression and risk of recurrence in women with DCIS regardless of the nuclear grade, type of treatment, age, and the cutoff value chosen. ${ }^{68,69}$ Rakovitch et $\mathrm{al}^{70}$ found that HER2overexpressing and Ki-67 high HG-DCIS was associated with an increased risk of recurrence regardless of nuclear grade and age. Balleine et $\mathrm{al}^{71}$ found that a combination of nuclear grade and Ki-67 was able to correctly classify DCIS into prognostic groups in $95.7 \%$ of cases. Unfortunately, lack of standardized methods and specific cut-off values limit the utility of Ki-67 in clinical practice. ${ }^{12}$

The TP53 tumor suppressor gene inactivating mutation is seen in $40 \%$ of HG-DCIS and has been associated with genomic instability and a worse outcome. ${ }^{72}$ Cyclooxygenase 2 (COX-2) is overexpressed in many human malignant tumors and has been linked to the process of carcinogenesis, cell survival, invasion, and metastasis. ${ }^{73,74}$ COX-2 catalyzes the synthesis of prostaglandins from arachidonic acid and is induced through inflammatory and mitogenic stimuli. ${ }^{74}$ de la Torre et $\mathrm{al}^{73}$ found increased COX-2 expression to be associated with higher Van Nuys prognostic grade. COX-2 overexpression in DCIS is also strongly correlated with HER2 overexpression, high proliferation rate, high nuclear grade, ER-negative status, and risk of recurrence. ${ }^{28,74,75} \mathrm{Bcl}-2$ is an apoptosis regulator and an independent prognostic marker in early phases of breast carcinoma. It is highly expressed in LG-DCIS; however, its expression gradually decreases as lesions become more aggressive. ${ }^{72}$ Androgen receptor is expressed in $30 \%$ to $40 \%$ of DCIS regardless of the grade. ${ }^{76}$ Most androgen receptor-positive cases are HG with apocrine features. GATA-3 mutations are more commonly seen in ER-positive DCIS. ${ }^{53} \mathrm{C}$-met increased levels are associated with vascular endothelial growth factor A and fibroblast growth factor receptor 1 expression, which contribute to angiogenesis. High expression of vascular endothelial growth factor A is correlated with biological aggressiveness of DCIS, whereas fibroblast growth factor receptor 1 amplification is correlated with progression to invasive cancer. ${ }^{72}$

The molecular signature of ER, PR, and bcl 2 negativity and high expression of $p 53$ and Ki-67 correlate with local recurrence. The p16, COX-2, and Ki-67 high expression is correlated with risk of progression to IBC. ${ }^{72}$

\section{Conclusions}

We reviewed the morphologic features of DCIS, major differential diagnoses, and pathologic information that need to be included in pathology reports to assist clinical decision making. With widespread use of mammography, the number of cases diagnosed as DCIS has significantly increased. 
Many of these lesions will never progress to invasive carcinoma. There is a need for identification of biomarkers that can stratify DCIS into different risk groups based on biology of the lesion because morphologic findings alone are not sufficient to separate lesions with potential to progression from lesions that do not progress. DCIS is extremely heterogeneous, further suggesting that classification based on morphologic finings may be an oversimplification. LG- and HG-DCIS have different patterns of molecular alterations. Characterization of DCIS on the molecular level may be able to better explain the variability in patient outcome and response to therapy. Unfortunately, despite intensive research, robust biomarkers that can predict risk of recurrence or progression of DCIS have not been discovered. In the future, most likely DCIS classification will include combining histologic findings with molecular profiling to improve risk stratification and to provide a personalized approach so patient management can be tailored to biological nature and malignant potential of the disease.

\section{References}

1. Sanati S, Warrick J, Hameed O, Allred DC: Breast pathology. Breast. Edited by Humphrey PA, Dehner LP, Pfeifer JD. In The Washington Manual of Surgical Pathology. ed 2. Philadelphia, PA: Lippincott Williams \& Wilkins, 2012. pp. 298-328

2. Ellis IO: Intraductal proliferative lesions of the breast: morphology, associated risk and molecular biology. Mod Pathol 2010, 23 Suppl 2:S1-S7

3. Morrow M, Schnitt SJ, Norton L: Current management of lesions associated with an increased risk of breast cancer. Nat Rev Clin Oncol 2015, 12:227-238

4. Carraro DM, Elias EV, Andrade VP: Ductal carcinoma in situ of the breast: morphological and molecular features implicated in progression. Biosci Rep 2014, 34:e00090

5. Ryan R, Tawfik O, Jensen RA, Anant S: Current approaches to diagnosis and treatment of ductal carcinoma in situ and future directions. Prog Mol Biol Transl Sci 2017, 151:33-80

6. Welch HG, Black WC: Using autopsy series to estimate the disease "reservoir" for ductal carcinoma in situ of the breast: how much more breast cancer can we find? Ann Intern Med 1997, 127:1023-1028

7. Toss M, Miligy I, Thompson AM, Khout H, Green AR, Ellis IO, Rakha EA: Current trials to reduce surgical intervention in ductal carcinoma in situ of the breast: critical review. Breast 2017, 35: 151-156

8. Masood S: Why the term 'low-grade ductal carcinoma in situ' should be changed to 'borderline breast disease': diagnostic and clinical implications. Womens Health (Lond) 2012, 8:57-62

9. Kanbayashi C, Iwata H: Current approach and future perspective for ductal carcinoma in situ of the breast. Jpn J Clin Oncol 2017, 47: $671-677$

10. Allegra CJ, Aberle DR, Ganschow P, Hahn SM, Lee CN, MillonUnderwood S, Pike MC, Reed SD, Saftlas AF, Scarvalone SA, Schwartz AM, Slomski C, Yothers G, Zon R: National Institutes of Health State-of-the-Science Conference statement: diagnosis and management of ductal carcinoma in situ September 22-24, 2009. J Natl Cancer Inst 2010, 102:161-169

11. Walker RA, Hanby A, Pinder SE, Thomas J, Ellis IO; National Coordinating Committee for Breast Pathology Research Subgroup: Current issues in diagnostic breast pathology. J Clin Pathol 2012, 65: $771-785$

12. Pang JM, Gorringe KL, Fox SB: Ductal carcinoma in situ - update on risk assessment and management. Histopathology 2016, 68:96-109
13. Allred DC, Wu Y, Mao S, Nagtegaal ID, Lee S, Perou CM, Mohsin SK, O'Connell P, Tsimelzon A, Medina D: Ductal carcinoma in situ and the emergence of diversity during breast cancer evolution. Clin Cancer Res 2008, 14:370-378

14. Collins LC: Precursor lesions of the low-grade breast neoplasia pathway. Surg Pathol Clin 2018, 11:177-197

15. Garijo MF, Val D, Val-Bernal JF: An overview of the pale and clear cells of the nipple epidermis. Histol Histopathol 2009, 24:367-376

16. Morrow M, Van Zee KJ, Solin LJ, Houssami N, ChavezMacGregor M, Harris JR, Horton J, Hwang S, Johnson PL, Marinovich ML, Schnitt SJ, Wapnir I, Moran MS: Society of Surgical Oncology-American Society for Radiation Oncology-American Society of Clinical Oncology Consensus Guideline on margins for breast-conserving surgery with whole-breast irradiation in ductal carcinoma in situ. Ann Surg Oncol 2016, 23:3801-3810

17. Benson JR, Wishart GC: Predictors of recurrence for ductal carcinoma in situ after breast-conserving surgery. Lancet Oncol 2013, 14: e348-e357

18. Ekatah GE, Turnbull AK, Arthur LM, Thomas J, Dodds C, Dixon JM: Margin width and local recurrence after breast conserving surgery for ductal carcinoma in situ. Eur J Surg Oncol 2017, 43: 2029-2035

19. Early Breast Cancer Trialists' Collaborative Group (EBCTCG), Correa C, McGale P, Taylor C, Wang Y, Clarke M, Davies C, Peto R, Bijker N, Solin L, Darby S: Overview of the randomized trials of radiotherapy in ductal carcinoma in situ of the breast. J Natl Cancer Inst Monogr 2010, 2010:162-177

20. Allred DC, Anderson SJ, Paik S, Wickerham DL, Nagtegaal ID, Swain SM, Mamounas EP, Julian TB, Geyer CE Jr, Costantino JP, Land SR, Wolmark N: Adjuvant tamoxifen reduces subsequent breast cancer in women with estrogen receptor-positive ductal carcinoma in situ: a study based on NSABP protocol B-24. J Clin Oncol 2012, 30: $1268-1273$

21. VandenBussche CJ, Elwood H, Cimino-Mathews A, Bittar Z, Illei PB, Warzecha HN: Clinicopathologic features of ductal carcinoma in situ in young women with an emphasis on molecular subtype. Hum Pathol 2013, 44:2487-2493

22. Wadsten C, Garmo H, Fredriksson I, Sund M, Wärnberg F: Risk of death from breast cancer after treatment for ductal carcinoma in situ. Br J Surg 2017, 104:1506-1513

23. Benson JR, Jatoi I, Toi M: Treatment of low-risk ductal carcinoma in situ: is nothing better than something? Lancet Oncol 2016, 17: e $442-\mathrm{e} 451$

24. Altintas S, Lambein K, Huizing MT, Braems G, Asjoe FT, Hellemans H, Van Marck E, Weyler J, Praet M, Van den Broecke R, Vermorken JB, Tjalma WA: Prognostic significance of oncogenic markers in ductal carcinoma in situ of the breast: a clinicopathologic study. Breast J 2009, 15:120-132

25. Punglia RS, Jiang W, Lipsitz SR, Hughes ME, Schnitt SJ, Hassett MJ, Nekhlyudov L, Achacoso N, Edge S, Javid SH, Niland JC, Theriault RL, Wong YN, Habel LA: Clinical risk score to predict likelihood of recurrence after ductal carcinoma in situ treated with breast-conserving surgery. Breast Cancer Res Treat 2018, 167: 751-759

26. Duggal S, Robin J, Julian TB: Ductal carcinoma in situ: an overview. Expert Rev Anticancer Ther 2013, 13:955-962

27. Ballehaninna UK, Chamberlain RS: Inclusion of tumor biology molecular markers to improve the ductal carcinoma in situ ipsilateral breast tumor recurrence nomogram predictability. J Clin Oncol 2011, 29:e97-e98

28. Mardekian SK, Bombonati A, Palazzo JP: Ductal carcinoma in situ of the breast: the importance of morphologic and molecular interactions. Hum Pathol 2016, 49:114-123

29. Martínez-Pérez C, Turnbull AK, Ekatah GE, Arthur LM, Sims AH, Thomas JS, Dixon JM: Current treatment trends and the need for better predictive tools in the management of ductal carcinoma in situ of the breast. Cancer Treat Rev 2017, 55:163-172 
30. Weigel MT, Dowsett M: Current and emerging biomarkers in breast cancer: prognosis and prediction. Endocr Relat Cancer 2010, 17: R245-R262

31. Hammond ME, Hayes DF, Wolff AC, Mangu PB, Temin S: American society of clinical oncology/college of American pathologists guideline recommendations for immunohistochemical testing of estrogen and progesterone receptors in breast cancer. J Oncol Pract 2010, 6:195-197

32. Sutela A, Vanninen R, Sudah M, Berg M, Kiviniemi V, Rummukainen J, Kataja V, Kärjä V: Surgical specimen can be replaced by core samples in assessment of ER, PR and HER-2 for invasive breast cancer. Acta Oncol 2008, 47:38-46

33. Arnedos M, Nerurkar A, Osin P, A'Hern R, Smith IE, Dowsett M: Discordance between core needle biopsy $(\mathrm{CNB})$ and excisional biopsy (EB) for estrogen receptor (ER), progesterone receptor $(\mathrm{PgR})$ and HER2 status in early breast cancer (EBC). Ann Oncol 2009, 20: $1948-1952$

34. Tamaki K, Sasano H, Ishida T, Miyashita M, Takeda M, Amari M, Tamaki N, Ohuchi N: Comparison of core needle biopsy (CNB) and surgical specimens for accurate preoperative evaluation of ER, PgR and HER2 status of breast cancer patients. Cancer Sci 2010, 101: 2074-2079

35. Lari SA, Kuerer HM: Biological markers in DCIS and risk of breast recurrence: a systematic review. J Cancer 2011, 2:232-261

36. Lazzeroni M, Dunn BK, Pruneri G, Jereczek-Fossa BA, Orecchia R, Bonanni B, DeCensi A: Adjuvant therapy in patients with ductal carcinoma in situ of the breast: the Pandora's box. Cancer Treat Rev 2017, 55:1-9

37. Van Bockstal M, Lambein K, Denys H, Braems G, Nuyts A, Van den Broecke R, Cocquyt V, De Wever O, Libbrecht L: Histopathological characterization of ductal carcinoma in situ (DCIS) of the breast according to HER2 amplification status and molecular subtype. Virchows Arch 2014, 465:275-289

38. Zaugg K, Bodis S: Is there a role for molecular prognostic factors in the clinical management of ductal carcinoma in situ (DCIS) of the breast? Radiother Oncol 2000 May, 55:95-99

39. Hendry S, Salgado R, Gevaert T, Russell PA, John T, Thapa B, et al: Assessing tumor-infiltrating lymphocytes in solid tumors: a practical review for pathologists and proposal for a standardized method from the International Immunooncology Biomarkers Working Group: part 1: assessing the host immune response, TILs in invasive breast carcinoma and ductal carcinoma in situ, metastatic tumor deposits and areas for further research. Adv Anat Pathol 2017, 24:235-251

40. Pruneri G, Lazzeroni M, Bagnardi V, Tiburzio GB, Rotmensz N, DeCensi A, Guerrieri-Gonzaga A, Vingiani A, Curigliano G, Zurrida S, Bassi F, Salgado R, Van den Eynden G, Loi S, Denkert C, Bonanni B, Viale G: The prevalence and clinical relevance of tumorinfiltrating lymphocytes (TILs) in ductal carcinoma in situ of the breast. Ann Oncol 2017, 28:321-328

41. Hendry S, Pang JB, Byrne DJ, Lakhani SR, Cummings MC, Campbell IG, Mann GB, Gorringe KL, Fox SB: Relationship of the breast ductal carcinoma in situ immune microenvironment with clinicopathological and genetic features. Clin Cancer Res 2017, 23:5210-5217

42. Van Bockstal M, Libbrecht L, Floris G, Lambein K, Pinder S: Stromal inflammation, necrosis and HER2 overexpression in ductal carcinoma in situ of the breast: another causality dilemma? Ann Oncol 2017, 28:2317

43. Toss MS, Miligy I, Al-Kawaz A, Alsleem M, Khout H, Rida PC, Aneja R, Green AR, Ellis IO, Rakha EA: Prognostic significance of tumor-infiltrating lymphocytes in ductal carcinoma in situ of the breast. Mod Pathol 2018, 31:1226-1236

44. Doebar SC, de Monyé C, Stoop H, Rothbarth J, Willemsen SP, van Deurzen CH: Ductal carcinoma in situ diagnosed by breast needle biopsy: predictors of invasion in the excision specimen. Breast 2016, 27:15-21

45. Beguinot M, Dauplat MM, Kwiatkowski F, Lebouedec G, Tixier L, Pomel C, Penault-Llorca F, Radosevic-Robin N: Analysis of tumour- infiltrating lymphocytes reveals two new biologically different subgroups of breast ductal carcinoma in situ. BMC Cancer 2018, 18:129

46. Abba MC, Gong T, Lu Y, Lee J, Zhong Y, Lacunza E, Butti M, Takata Y, Gaddis S, Shen J, Estecio MR, Sahin AA, Aldaz CM: A molecular portrait of high-grade ductal carcinoma in situ. Cancer Res 2015, 75:3980-3990

47. Agahozo MC, Hammerl D, Debets R, Kok M, van Deurzen CHM: Tumor-infiltrating lymphocytes and ductal carcinoma in situ of the breast: friends or foes? Mod Pathol 2018, 31:1012-1025

48. Wellings SR, Jensen HM: On the origin and progression of ductal carcinoma in the human breast. J Natl Cancer Inst 1973, 50: $1111-1118$

49. Moelans CB, de Wegers RA, Monsuurs HN, Maess AH, van Diest PJ: Molecular differences between ductal carcinoma in situ and adjacent invasive breast carcinoma: a multiplex ligation-dependent probe amplification study. Cell Oncol (Dordr) 2011, 34:475-482

50. Ellsworth RE, Ellsworth DL, Love B, Patney HL, Hoffman LR, Kane J, Hooke JA, Shriver CD: Correlation of levels and patterns of genomic instability with histological grading of DCIS. Ann Surg Oncol 2007, 14:3070-3077

51. Simpson PT, Reis-Filho JS, Gale T, Lakhani SR: Molecular evolution of breast cancer. J Pathol 2005, 205:248-254

52. Hannemann J, Velds A, Halfwerk JB, Kreike B, Peterse JL, van de Vijver MJ: Classification of ductal carcinoma in situ by gene expression profiling. Breast Cancer Res 2006, 8:R61

53. Pang JB, Savas P, Fellowes AP, Mir Arnau G, Kader T, Vedururu R, Hewitt C, Takano EA, Byrne DJ, Choong DY, Millar EK, Lee CS, O’Toole SA, Lakhani SR, Cummings MC, Mann GB, Campbell IG, Dobrovic A, Loi S, Gorringe KL, Fox SB: Breast ductal carcinoma in situ carry mutational driver events representative of invasive breast cancer. Mod Pathol 2017, 30:952-963

54. Vincent-Salomon A, Lucchesi C, Gruel N, Raynal V, Pierron G, Goudefroye R, Reyal F, Radvanyi F, Salmon R, Thiery JP, SastreGarau X, Sigal-Zafrani B, Fourquet A, Delattre O; Breast Cancer Study Group of the Institut Curie: Integrated genomic and transcriptomic analysis of ductal carcinoma in situ of the breast. Clin Cancer Res 2008, 14:1956-1965

55. Castro NP, Osório CA, Torres C, Bastos EP, Mourão-Neto M, Soares FA, Brentani HP, Carraro DM: Evidence that molecular changes in cells occur before morphological alterations during the progression of breast ductal carcinoma. Breast Cancer Res 2008, 10: R87

56. Sanati S, Allred DC: The progression of pre-invasive to invasive cancer. Edited by Fitzgerald R. In Pre-Invasive Disease: Pathogenesis and Clinical Management. New York, NY: Springer Science and Business Media, 2011. pp. 65-76

57. King TA, Sakr RA, Muhsen S, Andrade VP, Giri D, Van Zee KJ, Morrow M: Is there a low-grade precursor pathway in breast cancer? Ann Surg Oncol 2012, 19:1115-1121

58. Gerdes MJ, Gökmen-Polar Y, Sui Y, Pang AS, LaPlante N, Harris AL, Tan PH, Ginty F, Badve SS: Single-cell heterogeneity in ductal carcinoma in situ of breast. Mod Pathol 2018, 31:406-417

59. Perou CM, Sørlie T, Eisen MB, van de Rijn M, Jeffrey SS, Rees CA, Pollack JR, Ross DT, Johnsen H, Akslen LA, Fluge O, Pergamenschikov A, Williams C, Zhu SX, Lønning PE, BørresenDale AL, Brown PO, Botstein D: Molecular portraits of human breast tumours. Nature 2000, 406:747-752

60. Makretsov NA, Huntsman DG, Nielsen TO, Yorida E, Peacock M, Cheang MC, Dunn SE, Hayes M, van de Rijn M, Bajdik C, Gilks CB Hierarchical clustering analysis of tissue microarray immunostaining data identifies prognostically significant groups of breast carcinoma. Clin Cancer Res 2004, 10:6143-6151

61. Meijnen P, Peterse JL, Antonini N, Rutgers EJ, van de Vijver MJ: Immunohistochemical categorization of ductal carcinoma in situ of the breast. Br J Cancer 2008, 98:137-142

62. Tamimi RM, Baer HJ, Marotti J, Galan M, Galaburda L, Fu Y, Deitz AC, Connolly JL, Schnitt SJ, Colditz GA, Collins LC: 
Comparison of molecular phenotypes of ductal carcinoma in situ and invasive breast cancer. Breast Cancer Res 2008, 10:R67

63. Han K, Nofech-Mozes S, Narod S, Hanna W, Vesprini D, Saskin R, Taylor C, Kong I, Paszat L, Rakovitch E: Expression of HER2neu in ductal carcinoma in situ is associated with local recurrence. Clin Oncol (R Coll Radiol) 2012, 24:183-189

64. Kuerer HM, Albarracin CT, Yang WT, Cardiff RD, Brewster AM, Symmans WF, Hylton NM, Middleton LP, Krishnamurthy S, Perkins GH, Babiera G, Edgerton ME, Czerniecki BJ, Arun BK, Hortobagyi GN: Ductal carcinoma in situ: state of the science and roadmap to advance the field. J Clin Oncol 2009, 27:279-288

65. Tang P, Wang X, Schiffhauer L, Wang J, Bourne P, Yang Q, Quinn A, Hajdu S: Expression patterns of ER-alpha, PR, HER-2/neu, and EGFR in different cell origin subtypes of high grade and non-high grade ductal carcinoma in situ. Ann Clin Lab Sci 2006, 36:137-143

66. Meattini I, Saieva C, Bastiani P, Martella F, Francolini G, Lo Russo M, Paoletti L, Doria M, Desideri I, Terziani F, De Luca Cardillo C, Bendinelli B, Ciabatti C, Muntoni C, Tinacci G, Nori J, Smith H, Brancato B, Galli L, Sanchez LJ, Casella D, Bernini M, Orzalesi L, Carta GA, Bianchi S, Rossi F, Livi L: Impact of hormonal status on outcome of ductal carcinoma in situ treated with breastconserving surgery plus radiotherapy: long-term experience from two large-institutional series. Breast 2017, 33:139-144

67. Williams KE, Barnes NL, Cramer A, Johnson R, Cheema K, Morris J, Howe M, Bundred NJ: Molecular phenotypes of DCIS predict overall and invasive recurrence. Ann Oncol 2015, 26:1019-1025

68. Poulakaki N, Makris GM, Papanota AM, Marineli F, Marinelis A, Battista MJ, Boehm D, Psyrri A, Sergentanis TN: Ki-67 expression as a factor predicting recurrence of ductal carcinoma in situ of the breast: a systematic review and meta-analysis. Clin Breast Cancer 2018, 18: $157-167$

69. Poulakaki N, Makris GM, Battista MJ, Böhm D, Petraki K, Bafaloukos D, Sergentanis TN, Siristatidis C, Chrelias C,
Papantoniou N: Hormonal receptor status, Ki-67 and HER2 expression: prognostic value in the recurrence of ductal carcinoma in situ of the breast? Breast 2016, 25:57-61

70. Rakovitch E, Nofech-Mozes S, Hanna W, Narod S, Thiruchelvam D, Saskin R, Spayne J, Taylor C, Paszat L: HER2/neu and Ki-67 expression predict non-invasive recurrence following breastconserving therapy for ductal carcinoma in situ. Br J Cancer 2012, 106:1160-1165

71. Balleine RL, Webster LR, Davis S, Salisbury EL, Palazzo JP, Schwartz GF, Cornfield DB, Walker RL, Byth K, Clarke CL, Meltzer PS: Molecular grading of ductal carcinoma in situ of the breast. Clin Cancer Res 2008, 14:8244-8252

72. Kaur H, Mao S, Shah S, Gorski DH, Krawetz SA, Sloane BF, Mattingly RR: Next-generation sequencing: a powerful tool for the discovery of molecular markers in breast ductal carcinoma in situ. Expert Rev Mol Diagn 2013, 13:151-165

73. de la Torre J, Sabadell MD, Rojo F, Lirola JL, Salicru S, Reventos J, Ramón y Cajal S, Xercavins J: Cyclo-oxygenase type 2 is dysregulated in breast ductal carcinoma in situ and correlates with poor outcome. Eur J Obstet Gynecol Reprod Biol 2010, 151:72-76

74. Perrone G, Santini D, Vincenzi B, Zagami M, La Cesa A, Bianchi A, Altomare V, Primavera A, Battista C, Vetrani A, Tonini G, Rabitti C: COX-2 expression in DCIS: correlation with VEGF, HER-2/neu, prognostic molecular markers and clinicopathological features. Histopathology $2005,46: 561-568$

75. Polyak K: Molecular markers for the diagnosis and management of ductal carcinoma in situ. J Natl Cancer Inst Monogr 2010, 2010: 210-213

76. Oshilaja O, Nomani L, Calhoun BC, Montero AJ, Sturgis CD: Androgen receptors in resected ductal carcinoma in situ of breast: novel insights with possible implications for testing and targeted endocrine chemoprevention trials. Appl Immunohistochem Mol Morphol 2018, [Epub ahead of print] doi:10.1097/PAI.0000000000000625 